\title{
Initial report on subtrigonal Botulinum toxin-A (BoNT-A) therapy for female patients with urethral instability - results of a pilot study
}

\begin{abstract}
Introduction: We present the results of a pilot study performed in 5 female patients with overactive bladder symptoms $(\mathrm{OAB})$, combined with urethral instability, treated with subtrigonal Botulinum toxin-A (BoNT-A) injections. Treatment modalities for $\mathrm{OAB}$ have in common that they interfere in any part of efferent and afferent signaling from the bladder to the brain, the difference is in the localization and extent of interference. We hypothesized that patients with $\mathrm{OAB}$ symptoms and UI could benefit more from a treatment with the mode of action at the urodynamically identified problem.
\end{abstract}

Methods: This is a retrospective description of the pilot study we performed. Four patients had a long history of refractory idiopathic OAB symptoms, in one patient the main complaint was painfull bladder syndrome. In all patients UI was demonstrated during filling cystometry. Treatment was started with subtrigonal injections of $10 \mathrm{cc}$ lidocaine $1 \%$. If $\mathrm{OAB}$ symtoms improved with more than $50 \%$, treatment continued with subtrigonal injections with BoNT-A.

Results: Four out of 5 patients $\mathrm{OAB}$ symptoms improved more than $50 \%$ after subtrigonal BoNT-A injections. UI disappeared in one patient after treatment. In three other patients, the maximum amplitude of the urethral pressure variations decreased from more than $40 \mathrm{~cm} \mathrm{H} 2 \mathrm{O}$ up to a maximum of $20 \mathrm{~cm} \mathrm{H2O}$. The most remarkable change was the improvement in first sensation of filling.

Conclusion: Chemical denervation by subtrigonal BoNT-A injections in patients with $\mathrm{OAB}$ combined with UI, resulted in improvement of refractory OAB symptoms in all patients with refractory $\mathrm{OAB}$ as main complaint in this study.

Keywords: urge urinary incontinence, urodynamics, urethral instability, botulinum a toxin, overactive bladder

\author{
Volume 6 Issue 2 - 2018
}

\author{
Kummeling MT,' Dolmans VE, ${ }^{2}$ van Uhm \\ JI, ${ }^{2}$ de Vries L, ${ }^{2}$ Groenendijk PM,' van \\ Koeveringe GA, ${ }^{3}$ Elzevier $\mathrm{HW}^{2}$ \\ 'Department of Urology, Haaglanden Medisch Centrum (HMC), \\ Netherlands \\ ${ }^{2}$ Department of Urology, Leiden University Medical Centre \\ (LUMC), Netherlands \\ ${ }^{3}$ Department of Urology, Maastricht University Medical Centre \\ (MUMC), Netherlands
}

\begin{abstract}
Correspondence: Kummeling MT, Department of Urology HMC, Bronovolaan 5, 2597 AX The Hague, The Netherlands, Tel 00-3।-88-9794|44, Email m.kummeling@haaglandenmc.nl
\end{abstract}

Received: March 19,2018 | Published: April 12, 2018
Abbreviation: OAB, overactive bladder syndrome; DO, detrusor over activity; UI, urethral instability; ICS, international continence society; ICIRS, international consultation on incontinence research Society; BPS, bladder pain syndrome; BoNT-A botulinum toxin-A; POP, pelvic organ prolaps; PFMT, pelvic floor muscle therapy; FSF, first sensation of filling; BC, bladder capacity; SNM, sacral neuromodulation therapy

\section{Introduction}

Overactive bladder syndrome $(\mathrm{OAB})$ is a common, often underdiagnosed and under-treated condition that significantly affects the quality of life of patients. ${ }^{1}$ Unfortunately, in large part of the patients the underlying etiology of $\mathrm{OAB}$ is unknown. Urodynamic evaluation can be performed to learn more of the underlying etiology, but unfortunately symptoms of $\mathrm{OAB}$ are also present in patients without urodynamic abnormalities. Therefore, patients with $\mathrm{OAB}$ are a very heterogeneous patient population for whom a standard treatment protocol is difficult to establish. The treatments offered to patients are usually by preference and experience of the treating physician and effectiveness is established by trial and error.

During filling cystometry of urodynamic evaluation for $\mathrm{OAB}$, detrusor over activity (DO) is often observed. Urethral pressure variations - referred to as urethral instability (UI) by the International Continence Society (ICS) have also been observed and associated with OAB. ${ }^{2,3}$ However, the clinical relevance of this phenomenon is still controversial. DO can be observed in patients suffering from $\mathrm{OAB}$, but also in asymptomatic patients. For urethral instability (UI), the presence of the phenomenon in asymptomatic volunteers was one of the major reasons to abandon this as a pathophysiological phenomenon. It never made it to stay a within the International Continence Society (ICS) terminology.

Urinary continence is maintained by a low pressure reservoir and adequate resting pressure in the urinary sphincter. Therefore, the scope of research on $\mathrm{OAB}$ should not be limited to (over) activity of detrusor muscle, but should also take urethral function in consideration. This opinion is shared by the International Consultation on Incontinence Research Society (ICIRS), who recommended new clinical research to be performed on urethral pressure variations. ${ }^{4}$ In a recent review was concluded that despite the poor methodological quality of the performed studies UPV may be regarded a - potentially pathophysiological entity of its own within cohorts of patients with OAB. ${ }^{5}$

Nowadays treatment options for OAB include lifestyle modifications, pelvic floor muscle exercises, pharmacotherapy with 
anticholinergics or beta- 3 receptor agonists, pudendal or sacral nerve stimulation and intravesical Botulinum toxin-A (BoNT-A) therapy. ${ }^{6}$ In the past, surgical bladder denervation has been performed as treatment modality for OAB. This was first described in 1935 by Richer ${ }^{7}$ and afterwards modified by several colleagues. ${ }^{7,8}$

All the above mentioned treatments have in common that they interfere in any part of efferent and afferent signaling from the bladder to the brain; the difference is in the localization and extent of interference. In the present day invasive surgical denervation is rarely performed. The signaling from the nerves is blocked by intravesical injections of Botulinum toxin $\mathrm{A}$, it is a chemical denervation. Botulinum toxin A treatment in OAB has evidence based good results, ${ }^{9}$ however there are still patients suffering from refractory OAB. The former surgical denervation was performed outside the bladder wall, and thus had a different location of action than the current chemical intravesical injections.

We hypothesized that patients with $\mathrm{OAB}$ symptoms and UI are more likely to benefit from a treatment with the mode of action at the urodynamically identified problem.

In this pilot study we present the clinical and urodynamic results of subtrigonal/paraurethral chemical denervation treatment with BoNT-A injections in 5 patients with refractory OAB symptoms and UI.

\section{Material and methods}

This is a retrospective description of the pilot study we performed in 5 female patients. All patients were referred to the Urology department of Leiden University Medical Center and received already several treatments for $\mathrm{OAB}$ without success. Four patients had a long history of refractory idiopathic OAB symptoms. One patient was diagnosed with Bladder Pain Syndrome (BPS). All patients were evaluated by an urodynamic study with urethral pressure measurement. All urodynamic studies demonstrated urethral pressure variations. All patients consented to paraurethral treatment with BoNT-A injections.

In the past, improvement of $\mathrm{OAB}$ after subtrigonal injections with local anesthetics was a positive predictive factor for success of surgical denervation. ${ }^{7}$ Prior to the surgical procedure, local instillation with xylocaine/adrenalin or bupivavcain solution was performed. First unilaterally, subtrigonal, into the anterior fornix lateral, one $\mathrm{cm}$ to the cervix and at a depth of about $3 \mathrm{~cm}$. When there was an improvement of compliance or DO on cystometry and post void residual volume was less then $150 \mathrm{ml}$, there was a favorable result and treatment was continued with surgery. Resection of nerves was performed unilaterally or bilaterally through an incision in the anterior vaginal wall. Therefore we started treatment with subtrigonal/paraurethral injections of $10 \mathrm{cc}$ lidocaine $1 \%$. The patient was positioned in dorsolithotomy position. After disinfection, a urinary catheter was introduced. Traction on the balloon of the catheter was used to localize the position of the bladder neck and the trigonum. $10 \mathrm{~mL}$ of lidocaine was injected paraurethral and subtrigonal using a 22 gauge spinal needle. The patients were contacted the following day by telephone to evaluate the efficacy of the injections. A subjective improvement of $50 \%$ or more in urgency and/ or urge urinary incontinence symptoms reported by the patient was considered as a positive response. If the response was positive, treatment was continued with injections BoNT-A, $100 \mathrm{IU}$ in $10 \mathrm{ml}$ solution of $\mathrm{NaCl} 0,9 \%$. A description of the patients is mentioned below.

\section{Patient I}

Female nulliparous patient of 57 years old referred with urge urinary incontinence. History of slow transit constipation and bowel outlet obstruction, abdominal uterus extirpation and PDDNOS. Patient was referred with refractory $\mathrm{OAB}$ with urinary incontinence. She had pelvic floor muscle therapy (PFMT) and anti-muscarinic therapy without effect. Physical examination demonstrated no pelvic organ prolaps (POP). Cystoscopy revealed no abnormalities. Urodynamic evaluation demonstrated bladder capacity $(\mathrm{BC})$ of $380 \mathrm{ml}$, no DO, normal compliance, high urethral pressures till $100 \mathrm{~cm} \mathrm{H}_{2} \mathrm{O}$ and urethral pressure variations. After repeated PFMT without success, treatment with alpha blocking agent was started, without success. She was treated with subtrigonal lidocaine injections, which improved her symptoms with more than $50 \%$. Treatment was continued with subtrigonal BoNTA injections.

\section{Patient 2}

Female nulliparous patient of 38 years old; History of fibromyalgia, patient was referred with $\mathrm{OAB}$. She already received anti-muscarinic treatment, PMFT and intravesical GAG-replacement therapy with our success. Physical examination demonstrated no pelvic organ prolaps (POP). Urinary test were clear. Cystoscopy revealed no intravesical abnormalities. Urethra was dilated with short relieve of symptoms (2 days). Bladder diary mentioned total volume $1120 \mathrm{ml}$. Urinary portions of 60-250ml. Daytime frequency of 7 times, 1 time nocturia. Urodynamic evaluation demonstrated BC of $329 \mathrm{ml}$, no DO. First sensation of filling (FSF) at $64 \mathrm{ml}$ with urethral pressure drop. High urethral pressures and urethral pressure variations (UI) of $>40$ $\mathrm{cm} \mathrm{H}_{2} \mathrm{O}$. She had a very good response after subtrigonal lidocaine injections, so treatment was continued with subtrigonal BoNT-Ainjections.

\section{Patient 3}

Female patient of 69 years old referred with urge urinary incontinence. History of Stamey procedure, TVT-O and later TVT. Physical examination demonstrated no pelvic organ prolaps (POP). Patient reported daytime frequency of 8 times and at night 1 time, volumes $75-200 \mathrm{ml}$. Three to four incontinence episodes per day. Cystoscopy demonstrated trabeculation. Urodynamic evaluation demonstrated BC of $407 \mathrm{ml}$, no DO, FSF at $199 \mathrm{ml}$ with high pressure drop of urethral pressure and observation of urinary incontinence at lowest pressure point. After successful treatment with lidocaine injections, treatment was continued with subtrigonal BoNT-A injections.

\section{Patient 4}

Female multiparous patient of 62 years old referred with mixed urinary incontinence. No relevant medical history. Physical examination demonstrated no pelvic organ prolaps (POP). Urinary test were clear. Bladder diary demonstrated daytime frequency of 12 times and at nighttime frequency of 3 times. 9-10 incontinence episodes per day. Cystoscopy demonstrated trabeculation. Urodynamic evaluation demonstrated $\mathrm{BC}$ of $337 \mathrm{ml}$, no DO, FSF at $116 \mathrm{ml}$. High urethral pressures till $180 \mathrm{~cm} \mathrm{H}_{2} \mathrm{O}$ and urethral pressure variations (UI) of $>40 \mathrm{~cm} \mathrm{H}_{2} \mathrm{O}$. Post void residual volume of $225 \mathrm{ml}$. She had a good response after subtrigonal lidocaine injections, so treatment was continued with subtrigonal BoNT-A-injections. 


\section{Patient 5}

Female patient of 62 years old referred with urethral- and bladder pain syndrome. History of fibromyalgia, chronic fatigue syndrome, cardiac arrhythmias and nocturnal enuresis in her childhood. Patient suffered from urethral pain since 2010, she was treated with alpha booking agents, anti-muscarinic therapy without any effect and local depomedrol application with partial response. Physical examination demonstrated no pelvic organ prolaps (POP) or other abnormalities. Urinary tests were clear. Her bladder diary showed daytime urinary frequency of 11 times with volumes of $300-450 \mathrm{ml}$. Nocturia 2-3 times. Cystoscopy revealed trabeculation. Urodynamic evaluation demonstrated bladder capacity $(\mathrm{BC})$ of $730 \mathrm{ml}$, no DO, normal compliance, FSF at $113 \mathrm{ml}$, high urethral pressures till $150 \mathrm{~cm} \mathrm{H}_{2} \mathrm{O}$ and urethral pressure variations of $>40 \mathrm{~cm} \mathrm{H}_{2} \mathrm{O}$. No post voids residual volume. Pain worsens after micturition. She had a positive response after subtrigonal lidocaine injections, so treatment was continued with subtrigonal BoNT-A-injections

\section{Results}

The urodynamic evaluations before and after the subtrigonal BoNT-A injections and the clinical efficacy were compared. In Tabel 1, results of pre- and post-treatment urodynamic results are summarized.

Tabel I Urodynamic Results

\begin{tabular}{|c|c|c|c|c|c|}
\hline & Capacity (ml) & FSF (ml) & DO & UI & Effect BoNT-A \\
\hline Patient I & & & & & Positive \\
\hline Pre BoNT-A & 216 & n.a & No & $>40 \mathrm{~cm} \mathrm{H}_{2} \mathrm{O}$ & \\
\hline Post BoNT-A & 478 & 278 & No & No & \\
\hline Patient 2 & & & & & Positive \\
\hline Pre BoNT-A & 407 & 199 & No & $>40 \mathrm{~cm} \mathrm{H}_{2} \mathrm{O}$ & \\
\hline Post BoNT-A & 687 & 613 & No & $\max 20 \mathrm{~cm} \mathrm{H}_{2} \mathrm{O}$ & \\
\hline Patient 3 & & & & & Positive \\
\hline Pre BoNT-A & 329 & 64 & No & $>40 \mathrm{~cm} \mathrm{H}_{2} \mathrm{O}$ & \\
\hline Post BoNT-A & 363 & 200 & No & $\max 20 \mathrm{~cm} \mathrm{H}_{2} \mathrm{O}$ & \\
\hline Patient 4 & & & & & Positive \\
\hline Pre BoNT-A & 337 & 116 & No & $>40 \mathrm{~cm} \mathrm{H}_{2} \mathrm{O}$ & \\
\hline Post BoNT-A & n.a. & n.a & No & & \\
\hline Patient 5 & & & & & Negative \\
\hline Pre BoNT-A & 730 & 113 & No & $>40 \mathrm{~cm} \mathrm{H}_{2} \mathrm{O}$ & \\
\hline Post BoNT-A & n.a & n.a. & No & & \\
\hline \multicolumn{6}{|l|}{ Average } \\
\hline Pre BoNT-A (pt I-5) & 403 & 123 & & & \\
\hline Post BoNT-A (pt I-3) & 509 & 363 & & & \\
\hline
\end{tabular}

\section{Patient I}

There was a good response to BoNT-A injections for four months. Urodynamic evaluation after the second series of injections demonstrated $\mathrm{BC}$ of $480 \mathrm{ml}$, no DO, still there was high urethral pressure around $100 \mathrm{~cm} \mathrm{H}_{2} \mathrm{O}$, but no pressure variations were seen. Treatment has been successfully repeated.

\section{Patient 2}

Urge complaints responded good on this treatment. Urodynamic evaluation after treatment demonstrated BC of $363 \mathrm{ml}$, no DO, FSF of $200 \mathrm{ml}$ and $114 \mathrm{ml}$. UI was still present, but with variations of maximal $20 \mathrm{~cm} \mathrm{H}_{2} \mathrm{O}$. Subtrigonal BoNT-A treatment has been repeated several times, with good response.

\section{Patient 3}

Patient had a good response after subtrigonal BoNT-A-injections. Urodynamic evaluation after BoNT-A treatment demonstrated BC of 687 and $756 \mathrm{ml}$, no DO, FSF at 613 and $645 \mathrm{ml}$ UI was observed, but with maximal $20 \mathrm{~cm} \mathrm{H}_{2} \mathrm{O}$ and not related to FSF.

\section{Patient 4}

Patient had a good response after subtrigonal BoNT-A-injections. Because of hip replacement surgery, post-treatment urodynamic evaluation has not been performed yet.

\section{Patient 5}

Patient had no positive response. After a few days she developed a urinary fact infection. After treating this infection complaints were there as worse as pre-treatment.

\section{Discussion}

In this heterogeneous group of patients with urethral instability (UI), we demonstrated an improvement of com-plaints in four out of five patients treated with subtrigonal BoNT-A injections. An interesting finding, apart from the increased bladder capacity, is the increased bladder volume at FSF.

Groenendijk, et al. ${ }^{10}$ concluded that presence of UI was a positive predictive factor for success of sacral neuromodulation therapy 
(SNM) for $\mathrm{OAB}$ and a better predictive factor then presence of $\mathrm{DO}$ in pretreatment urodynamic evaluation. Patients with UI responded well on SNM, however, UI was still present in post treatment urodynamic evaluation. Consistent with these findings, in our study, urethral pressure variations were still present in post-treatment urodynamic evaluation, but with a decreased amplitude of variations. 60 years ago, in the time of Ingelman-Sundberg, the group of 34 patients treated with surgical denervation was already split into 6 different categories, indicating how heterogeneous patient population with $\mathrm{OAB}$ is and has been over the years. Thirty patients were cured of their symptoms, with mean follow up of seven years. ${ }^{7}$ At that time, changes in cystometry and compliance of the bladder were observed. Urethral pressures were not measured in those days.

Classification based on clinical and urodynamic characteristics of $\mathrm{OAB}$ patients would contribute to targeted therapy. To debate is the question whether $\mathrm{OAB}$ is from urodynamic point of view a bladderonly problem or not. In the recent decades, urodynamic evaluations of patients have been further developed. An effort has been made to define what normal or physiological observations are and when to speak of abnormal or pathophysiological findings. However, the bladder has been the center of attention. If assumed that the feeling of imminent micturition has its origins in the urethra, ${ }^{11}$ intervening in afferent sensory input from the urethra could have a positive effect on $\mathrm{OAB}$ symptoms of patients. It might be that efferent motor response is being of lesser importance. Apparently, sensory input is influenced by neuromodulation and surgical or chemical denervation.

Local injections with lidocaine temporarily and reversibly prevent the formation and transmission of stimuli along the peripheral nervous pathways and nerve endings, creating a temporary numbness. BoNT-A results in inhibition of exocytosis of acetylcholine containing vesicles at the motor endplate, making transmission of the nerve impulse to the motor endplate of the muscle impossible and resulting in a weak paralysis. Despite the differences in mechanism of action of BoNT-A and lidocaine, local injections with lidocaine have been of predictive value in regard to response on BoNT-A treatment. ${ }^{7,8}$

Probably, we should see $\mathrm{OAB}$ as a form of neuropathy or bladder nerve supply dysfunction, which has different forms of expression. Depending on the affected branches, different complaints will be reported as major complaint of the patients, resulting in a heterogenous population with multiple complaints within pudendal nerve supply area.

\section{Conclusion}

Chemical denervation by subtrigonal BoNT-A injections resulted in improvement of refractory $\mathrm{OAB}$ symptoms in four out of five patients in this small pilot study. The largest improvement was in FSF. Further prospective research is necessary to define the condition of $\mathrm{UI}$ in patient with $\mathrm{OAB}$. Besides, new treatment modalities like chemical denervation with subtrigonal BoNT-A injections should be explored by correlation of bladder diaries, validated questionnaires and urodynamic evaluations, with special attention for FSF.

\section{Acknowledgements}

There is no financial assistance for the research work. Research was initiated by HW Elzevier, staff member / consultant Urology in Leiden University Medical Centre.

\section{Conflict of interest}

Authors declare there is no conflict of interest in publishing the article.

\section{References}

1. Coyne KS, Sexton CC, Vats V, et al. National community prevalence of overactive bladder in the United States stratified by sex and age. Urology. 2011;77(5):1081-7.

2. Wise BG, Cardozo LD, Cutner A, et al. Prevalence and significance of urethral instability in women with detrusor instability. $\mathrm{Br} J$ Urol. 1993;72(1):26-9.

3. McLennan MT, Melick C, Bent AE. Urethral instability: clinical and urodynamic characteristics. Neurourol Urodyn. 2001;20(6):653-60.

4. Gammie A, Bosch R, Djurhuus JC, et al. Do we need better methods of assessing urethral function: ICI-RS 2013? Neurourol Urodyn. 2014;33(5):587-90.

5. Kummeling MT, Rosier PF, Elzevier HW, et al. Continuous urethral pressure measurements; measurement techniques; pressure variations; clinical interpretations; and clinical relevance. A Systematic Literature Analysis. Neurourol Urodyn. 2017;36(1):51-6.

6. Allahdin S, Oo N. An overview of treatment of overactive bladder syndrome in women. J Obstet Gynaecol. 2012;32(3):217-21.

7. Ingelman Sundberg A. Partial denervation of the bladder, A new operation for the treatment of urge incontinence and similar conditions in women. Acta Obstet Gynecol Scand. 1959;38:487-502.

8. Cespedes RD, Cross CA, McGuire EJ. Modified Ingelman-Sundberg bladder denervation procedure for intractable urge incontinence. $J$ Urol. 1996;156(5):1744-7.

9. Anger JT, Weinberg A, Suttorp MJ, et al. Outcomes of intravesical botulinum toxin for idiopathic overactive bladder symptoms: a systematic review of the literature. $J$ Urol. 2010;183(6):2258-64.

10. Groenendijk PM, Heesakkers JP, Lycklama A Nijeholt AA. Urethral instability and sacral nerve stimulation-a better parameter to predict efficacy? J Urol. 2007;178(2):568-72.

11. Andersson KE. Bladder activation: afferent mechanisms. Urology. 2002;59(5 Suppl 1):43-50. 